\title{
Spectroscopic characterization of the on-surface induced (cyclo)dehydrogenation of a N-heteroaromatic compound on noble metal surfaces
}

\author{
I. Palacio, ${ }^{a *}$ A. L. Pinardi, ${ }^{a}$ J. I. Martínez, ${ }^{a}$ A. Preobrajenski, ${ }^{b}$ A. Cossaro,,${ }^{c}$ A. Jancarik, ${ }^{d}$ I. Stará, ${ }^{d}$ I. Starý, ${ }^{d}$ \\ J. Méndez, ${ }^{a}$ J. A. Martín-Gago, ${ }^{a}$ M. F. López ${ }^{a}$
}

\begin{abstract}
New nanoarchitectures can be built from polycyclic aromatic hydrocarbons (PAHs) by exploiting the capability of some metal surfaces of inducing cyclodehydrogenation reactions. This bottom-up approach allows the formation of nanostructures with different dimensionality from the same precursor as a consequence of the diffusion and coupling of the PAHs adsorbed on the surface. In this work we present a thorough study, by means of a combination of X-ray Photoemission Spectroscopy, Near-Edge X-ray Absorption Fine Structure and Scanning Tunneling Microscopy with first principle calculations, of the structural and chemical transformations undergone by pyridyl-substituted dibenzo[5]helicene on three coinage surfaces, namely $\mathrm{Cu}(110), \mathrm{Cu}(111)$ and $\mathrm{Au}(111)$. Upon annealing, on-surface chemical reactions are promoted affecting the adsorbate/substrate and the molecule/molecule interactions. This thermally induced process favours the transformation from diffusing isolated molecules to polymeric nanographene chains and finally to $\mathrm{N}$-doped graphene.
\end{abstract}

\section{Introduction}

New organic materials have the potential of substituting silicon-based technology in electronic devices, and are already widely spread in organic light emitting diodes (OLED) and in new designs of solar cells. Surface-mediated chemical modification of polycyclic aromatic hydrocarbons (PAHs), emerges as an excellent way to synthesize novel nanostructures, with a precise control of their composition and of their electronic properties. Moreover, this bottom-up approach may allow controlling a specific reaction path at the atomic level, leading to tailored reaction outcomes. In particular, thermally induced (cyclo)dehydrogenation (and/or dehalogenation) of PAHs followed by covalent coupling on transition metal surfaces may result in the synthesis of new zero-, one- and two-dimensional nanoarchitectures. ${ }^{1-6}$

In the field of on-surface synthesis of new nano-objects from their functional molecular building blocks, the selection of a correctly designed precursor is vital to achieve a desired outcome. In particular, the electronic doping of the resultant structure can be controlled by introducing foreign atoms into the precursor. For instance, the pyridyl-substituted dibenzo[5]helicene (called DiPy[5]DBH in the following) can hierarchically form $\mathrm{N}$-doped nanographene or nano-domes units upon annealing on $\mathrm{Pt}(111) .{ }^{7}$ Other complex molecules can lead to the formation of specific structures as graphene nanoribbons once the correct precursor and surface are chosen. ${ }^{6}$

On top of that, the choice of the substrate is also a crucial parameter. Indeed, our group has shown that the diffusion of the $\mathrm{PAH}$ precursor on the surface defines the dimensionality of the reaction products. ${ }^{1}$ On one hand, a highly reactive surface such as $\mathrm{Pt}(111)$ does not allow surface diffusion, impeaching the interaction between neighbouring molecules, so that thermal activation results in the intramolecular cyclodehydrogenation and the formation of new zerodimensional objects. On the other hand, when a PAH is deposited on a weakly reactive surface such as $A u(111)$, the molecules diffuse at room temperature; upon annealing, they undergo cyclodehydrogenation and dehydrogenative polymerization, and as a consequence, twodimensional polymeric chains form since the diffusion allows the molecules to meet and react with each other. Other coinage metal surfaces such as $\mathrm{Cu}(110)$ and $\mathrm{Cu}(111)$ also act as catalysts towards the formation of polymers since they promote diffusion. In particular, in ref. [1] we demonstrated that the structures formed on different coinage metal surfaces from the same 
$\mathrm{PAH}$ (namely DiPy[5]DBH) are analogous, but their relative formation temperatures strongly depend on the substrate.

Most of the works published until now in this field use Scanning Tunnelling Microscopy (STM) or non-contact Atomic Force Microscopy (nc-AFM) ${ }^{8}$ images to identify both, precursors and reaction products, while spectroscopic characterization of the processes is less common. On the other hand, core-level electron spectroscopies allow following details and exploring reaction paths that cannot be inferred by the analysis of STM/AFM images. In this work, we study in detail the adsorption of DiPy[5]DBH on three different coinage metal surfaces, namely $\mathrm{Cu}(110), \mathrm{Cu}(111)$ and $A u(111)$, and follow surface induced chemical reactions as a function of the substrate temperature. We used a combination of Scanning Tunneling Microscopy (STM), Near-Edge Xray Absorption Fine Structure (NEXAFS) and X-ray Photoemission Spectroscopy (XPS) to characterize the as-deposited molecules and their (cyclo)dehydrogenative polymerization upon annealing. Importantly, the presence of substitutional nitrogen in the precursor leads to the formation of $\mathrm{N}$-doped nanostructures. As analologous results have been found for the tree systems under study we show in this publication the most representative ones. Additionally, to shed light on the role of the molecular diffusion in the formation of novel nanoarchitectures we have designed a heuristic theoretical procedure. This approach allows to monitor the energetics and kinetic barriers of the fusion of the cycles of two partially dehydrogenated diffusing DiPy[5]DBH molecules towards their full linkage on the $\mathrm{Cu}(110), \mathrm{Cu}(111)$ and $\mathrm{Au}(111)$ surfaces following the minimum energy path (MEP). For that purpose we have calculated formation enthalpies $(\mathrm{H})$ and barrier heights along the MEP at the transition state (TS), $\triangle \mathrm{E}$, within a density functional theory (DFT)-based implementation of the climbing-image nudge elastic band (Cl-NEB) framework as described below.

\section{Experimental Section}

DiPy[5]DBH was synthesized according to the procedure described elswhere. ${ }^{1}$ The experiments have been carried out in three ultra-high vacuum (UHV) chambers, all with a base pressure of $1 \times 10^{-10}$ mbar. In all of them, the molecular powder was sublimated from a homemade Ta crucible annealed at $575 \mathrm{~K}$ controlled by a type-K thermocouple spot-welded to it. The metal samples were cleaned with cycles of sputtering $\left(\mathrm{Ar}^{+}\right.$pressure of $\left.1 \times 10^{-6} \mathrm{mbar}\right)$ and annealing $(800 \mathrm{~K})$, and the molecules were deposited on the clean samples kept at room temperature. NEXAFS and XPS have been recorded at the D1011 beamline at MAX-Lab (Lund, Sweden) and at ALOISA beamline $^{9}$ at Elettra (Trieste, Italy). They provide precise chemical information of the surface reactions, and are highly useful to understand the mechanisms of (cyclo)dehydrogenation in an atomistic way. These results are complemented with room temperature (RT) STM images (Omicron), which provide structural information.

The photon energy for measuring the N1s and C1s signals was $480 \mathrm{eV}$ and $380 \mathrm{eV}$ respectively. The intensity of both N1s and C1s peaks has been normalised to the same height. The peaks were fitted with a Lorentzian width of $180 \mathrm{meV}$ convoluted with a Gaussian component of about $600 \mathrm{meV}$, and by minimizing the number of components for each peak. NEXAFS spectra were measured with linearly polarized light and a photon energy resolution of $80 \mathrm{meV}$. The sample was held at room temperature, and spectra were recorded by changing the photon incidence angle $\left(\mathrm{P}\right.$-polarization $=20^{\circ}$ and $\mathrm{S}$-polarization $\left.=90^{\circ}\right)$.

\section{Theoretical Section}

Computational methods. In the ab initio atomistic simulations, total energies, forces, and stresses were minimized by using DFT as implemented in the plane-waves package QUANTUM ESPRESSO. ${ }^{10}$ DFT+D2 method of Grimme has been used to add dispersive forces to conventional density functionals. ${ }^{11,12}$ Ion-electron interaction is modelled by ultrasoft pseudopotentials, ${ }^{13}$ and exchange-correlation (XC) effects are treated by the PBE generalizedgradient approximation (GGA). ${ }^{14}$ All the Brillouin zones were discretized by optimal MonkhorstPack grids, ${ }^{15}$ and an energy cut-off of $500 \mathrm{eV}$ yields total energies within an accuracy of \pm 0.05 $\mathrm{eV}{ }^{16}$ Residual atomic forces and lattice stresses were lower than $0.01 \mathrm{eV} / \AA$ and $0.1 \mathrm{GPa}$, respectively. Transition states (TSs) have been investigated here within the Climbing-image Nudged Elastic Band (CI-NEB) approach. ${ }^{10,17-19}$ 
Models. The system geometries considered in the calculations for the $\mathrm{Cu}(110), \mathrm{Cu}(111)$ and $\mathrm{Au}(111)$ metal surfaces consist in: i) slabs of four physical layers (minimum perpendicular distance of $\sim 25 \AA$ of vacuum between neighbouring cells); and ii) full periodic boundary conditions representing infinite metal surfaces. Each substrate metal layer contained 90 atoms for $\mathrm{Cu}(110)$, and 96 for $\mathrm{Cu}(111)$ and $\mathrm{Au}(111)$, with unit cells of size $(21.6 \times 38.2) \AA^{2},(27.1 \times$ $20.9) \AA^{2}$ and $(23.4 \times 35.0) \AA^{2}$, respectively. Each unit cell included two partially dehydrogenated DiPy[5]DBH molecules (with a lack of three hydrogen atoms each) located as initial state at a short distance and with their corresponding dehydrogenated parts facing each other favouring the linking trajectory. Before locating the partially dehydrogenated molecules on the surfaces, and in order to speed up the whole structural relaxations, the gas-phase geometry of the molecule has been pre-optimized. Only the two bottom physical metal layers were fixed during the calculation of the MEPs. As a test, an additional metal layer was included for some intermediate images for the case of the $A u(111)$ to check the convergence degree in the energetics of the system, with no significant variation w.r.t. the four-layers case.

\section{Results and Discussion}

Figure 1a exhibits a ball-and-stick model of the precursor with white, light-blue and dark-blue spheres representing $\mathrm{H}, \mathrm{C}$ and $\mathrm{N}$ atoms, respectively. Figures $1 \mathrm{~b}$-d show STM images of the evolution of $0.5 \mathrm{ML}$ of $\mathrm{DiPy}[5] \mathrm{DBH}$ deposited on $\mathrm{Cu}(110)$ as the substrate temperature is increased. At RT (Figure 1b), the deposited molecules are in a physisorbed state and diffuse faster than the scanning speed, and are, therefore, invisible to the STM. After annealing the sample at $560 \mathrm{~K}$, the molecules start to partially undergo dehydrogenative polymerisation, and to react with each other to form dimers, trimers or very small oligomers (Figure 1c). However, even after annealing at this temperature, some molecules are still diffusing: indeed, the strokes along scanning direction in the images correspond to moving molecules. Further annealing at about $680 \mathrm{~K}$ induces further dehydrogenation and the plane rings from different molecules fuse to form static polymers on the surface (Figure 1d).

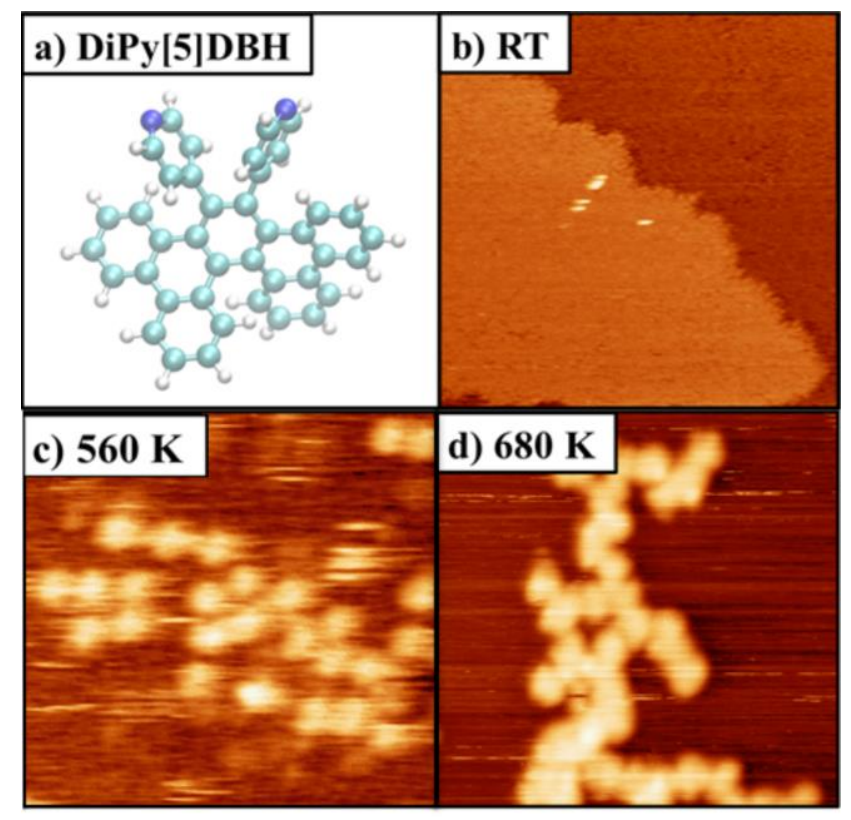

Figure 1. Ball-and-stick model of the precursor DiPy[5]DBH. b-d) STM images of the evolution of DiPy[5]DBH on Cu(110) for different temperatures. b) The precursor diffusing at $R T$ is not visible $(40 \times 40$ $\mathrm{nm}^{2} ;$ Vsample $\left.=1.0 \mathrm{~V}\right)$. c) The diffusing molecules coexist with dimers or trimers at $560 \mathrm{~K}\left(12.5 \times 12.5 \mathrm{~nm}^{2}\right.$; Vsample $=-2.0 \mathrm{~V}$ ). d) The precursor rings fuse between them and form single-molecule polymers at $680 \mathrm{~K}$ $\left(12.5 \times 12.5 \mathrm{~nm}^{2} ;\right.$ Vsample $\left.=2.0 \mathrm{~V}\right)$. 
At this stage, the individual molecules forming building blocks of the linked chains can be clearly distinguished, and their size (diameter $\sim 1.2 \mathrm{~nm})$ and apparent height $(\sim 0.22 \mathrm{~nm})$ is similar to the $\mathrm{N}$-doped nanographene found on $\mathrm{Pt}(111){ }^{7}$ Additional annealing of the sample leads to a decomposition of the molecular chains, and from $750 \mathrm{~K}$ graphene submonolayer patches begin to form. Analogous molecular transformations were observed on $\mathrm{Cu}(111)$ and on $\mathrm{Au}(111) 1$ although at slightly different temperature values. For the case of $\mathrm{Au}(111)$, graphene regions were never observed upon annealing in our experiments since we avoided high temperatures to prevent damaging the sample.

In order to gain better insights into the nature of adsorbate/substrate interactions NEXAFS and XPS measurements were performed. Specifically, the C K-edge NEXAFS and the N1s and C1s XPS of the adsorbed molecules provide very detailed information on the chemical sate of adsorbing atoms, and additionally NEXAFS provides information about the orientation angle of the $\mathrm{PAH}$ relative to the surface. Figure $2 \mathrm{a}$ shows the room temperature partial electron yield
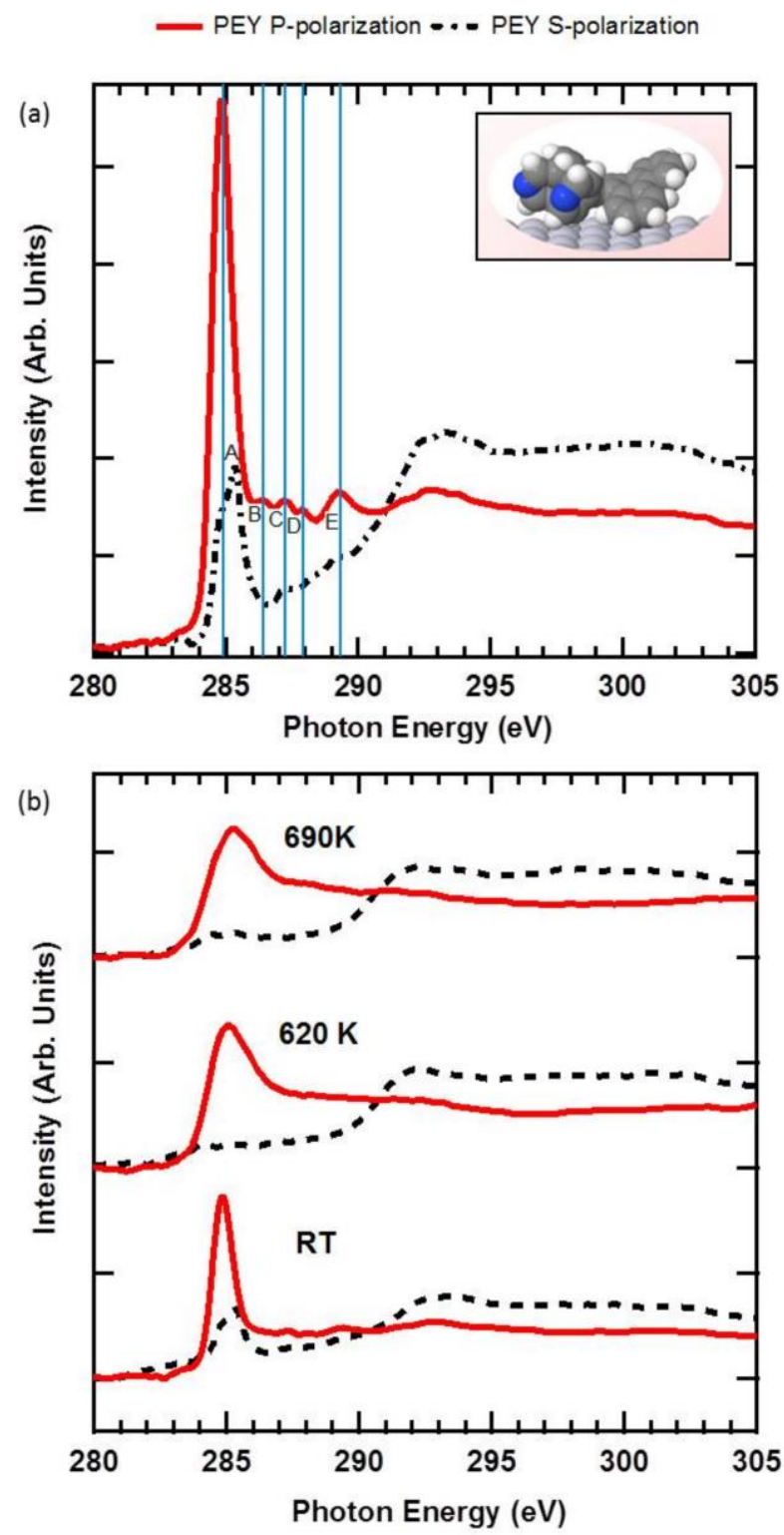

Figure 2. a) PEY C K-edge NEXAFS of one monolayer of DiPy[5]DBH/Cu(111) at RT recorded for both $p$ polarised (red solid curve) and s-polarised (black dashed curve) light. The blue lines indicate the position of the five peaks A-E; the inset exhibits the three-dimensional nature of the as-deposited molecule. b) Evolution of the NEXAFS from RT to 690K. 
(PEY) C K-edge normalized NEXAFS spectra of DiPy[5]DBH/Cu(111) for one monolayer coverage, and the evolution of the spectra from RT to $690 \mathrm{~K}(\mathrm{~b})$. The spectra were recorded with both s-polarised light (electric field being parallel to the surface, dashed black curves) and $p$ polarised light (electric field being perpendicular to the surface, solid red curves).

In general, the energy range of absorption of the $\pi^{*}$ states of the aromatic rings lies between 284-290 eV, and above $290 \mathrm{eV}$ we detect the broad continuum of $\sigma^{*}$ resonances. In the $\pi^{*}$ region, we observe five peaks, denoted as $A, B, C, D$ and $E$. The most intense peaks at 284.9 and $285.3 \mathrm{eV}$ are due to de C1s transitions to the LUMO $\pi^{*}$ states ${ }^{20-23}$ of non-equivalent $\mathrm{C}$ sites in the molecules. ${ }^{24,25}$ There is a large dichroism between the $s$ - and $p$-polarised signals, even though the $s$-polarised signal does not vanish. The $\pi^{*}$ region is less intense for $s$-polarised light and the $\sigma^{*}$ region is less intense for $p$-polarises light. This means that the molecules are not completely flat because they conserve the three dimensional structure of an intact free molecule (see inset of Figure 2a). However, the s-polarised intensity is smaller than the p- one, so the benzene rings of the molecules are laying on the surface at an average angle lower than 45은 as they try to couple their aromatic rings parallel to the surface. Moreover, for p-polarised light, the peak at $284.9 \mathrm{eV}$ is the most intense; while for s-polarised light the one at $285.3 \mathrm{eV}$ is prominent. This asymmetry suggests again that the as-deposited diffusing molecule is three-dimensional since the contribution to the LUMO of the C1s electrons of carbon atoms in two different positions is geometrically different. In previous literature, the less intense peaks $\mathrm{B}(286.4 \mathrm{eV}), \mathrm{C}$ $(287.3 \mathrm{eV}), \mathrm{D}(288.0 \mathrm{eV})$ and $\mathrm{E}(289.4 \mathrm{eV})$ have been assigned differently, with peaks B-D often associated with transitions of the $\mathrm{C} 1 \mathrm{~s}$ electrons into $\sigma^{*}$ orbitals located on $\mathrm{C}-\mathrm{H}$ bonds ${ }^{21,23,24,26-28}$ with an admixture of Rydberg 3 s states, ${ }^{29,30}$ while peak $E$ can be a signature of the second $\pi^{*}$ resonance in simple aromatic hydrocarbons ${ }^{31,21}$. For all these features we observe an anisotropy corresponding to a perpendicular bond. It is not surprising that the feature $\mathrm{B}-\mathrm{C}$ is split, as the transitions to the $\sigma^{*}(\mathrm{C}-\mathrm{H})$ states can occur on $\mathrm{C}$ atoms with slightly varying chemical state, including those in the pyridine rings. The broad features above $291 \mathrm{eV}$ represent $\mathrm{C} 1 \mathrm{~s}$ transitions to the $\sigma^{*}(\mathrm{C}-\mathrm{C})$ orbitals. ${ }^{21,22,32}$ The inset of Figure 2 a shows the DFT calculated adsorption geometry of the molecule adsorbed on the surface, which maintains its three-dimensional character. Figure $2 \mathrm{~b}$ shows the evolution of the NEXAFS from room temperature to $690 \mathrm{~K}$. The main peak at $284.9 \mathrm{eV}$ widens and the peaks in the $\mathrm{C}-\mathrm{H}$ region fade away, which suggests that the molecule dehydrogenates and hybridises with the surface upon annealing. The broadening of peak $A$ with temperature indicates the formation of graphene-like structures and a higher chemical interaction with the metal substrate. ${ }^{22,26}$ Importantly, the strong dichroism of the $\pi^{*}$ contribution of the NEXAFS spectrum for the two ( $s$ - and $\mathrm{p}-$ ) polarizations at $620 \mathrm{~K}$ indicates that the molecule flattens due to intramolecular cyclodehydrogenation as a consequence of the formation of new fused bonds. Due to steric constraints, the only way for the pristine molecules to flatten is by cyclodehydrogenation. Hence, coupled molecules, oligomers and polymeric networks are formed by dehydrogenative polymerisation as the temperature increases.

XPS spectra complement the NEXAFS and the STM data by giving insight into the chemical state of the molecule. Besides, the XPS of the sample annealed in steps is crucial to understand the chemical behaviour of the molecule upon (cyclo)dehydrogenation. Figure 3 shows the C1s (right) and the N1s (left) XPS peaks of submonolayer coverage of $\mathrm{DiPy}[5] \mathrm{DBH} / \mathrm{Cu}(110)$ at different temperatures. At room temperature we can distinguish up to three components in the C1s XPS signal: C1, the main one at $285.2 \mathrm{eV}$ (light blue), C2 at $284.6 \mathrm{eV}$ (light green), C3 at $285.9 \mathrm{eV}$ (purple). From the STM and the NEXAFS we know that at room temperature the molecules are diffusing and are not completely flat because of their three-dimensional structure. The presence of such a high number of peaks indicates the complexity of the free, low interacting molecule, with the $\mathrm{C}$ atoms existing in different chemical molecular environments. C1s peak transforms upon annealing becoming narrower as it loses components (or they become less important). The main carbon component ( $\mathrm{C} 1$ light blue) with a binding energy of $285.2 \mathrm{eV}$ is the most intense peak at room temperature and throughout the whole annealing series. In fact, its relative importance increases with temperature. It represents the $\mathrm{C}-\mathrm{C}$ in the $\mathrm{sp}^{2}$ 
configuration..$^{20,33-37}$ Upon annealing, each component of the XPS spectra undergoes a binding energy shift towards low values. This is indicative of the emerging interaction between molecules and surface after thermal treatment, with a net charge transfer from the surface to the molecule. The light green (C2) peak is ascribed to the $\mathrm{sp}^{2} \mathrm{C}$ bonded to two $\mathrm{C}$ atoms and one $\mathrm{H}$ atom and its relative spectral weight decreases with temperature. ${ }^{33,34,36}$ This result is expected as a consequence of both cyclodehydrogenation and dehydrogenative polymerisations.

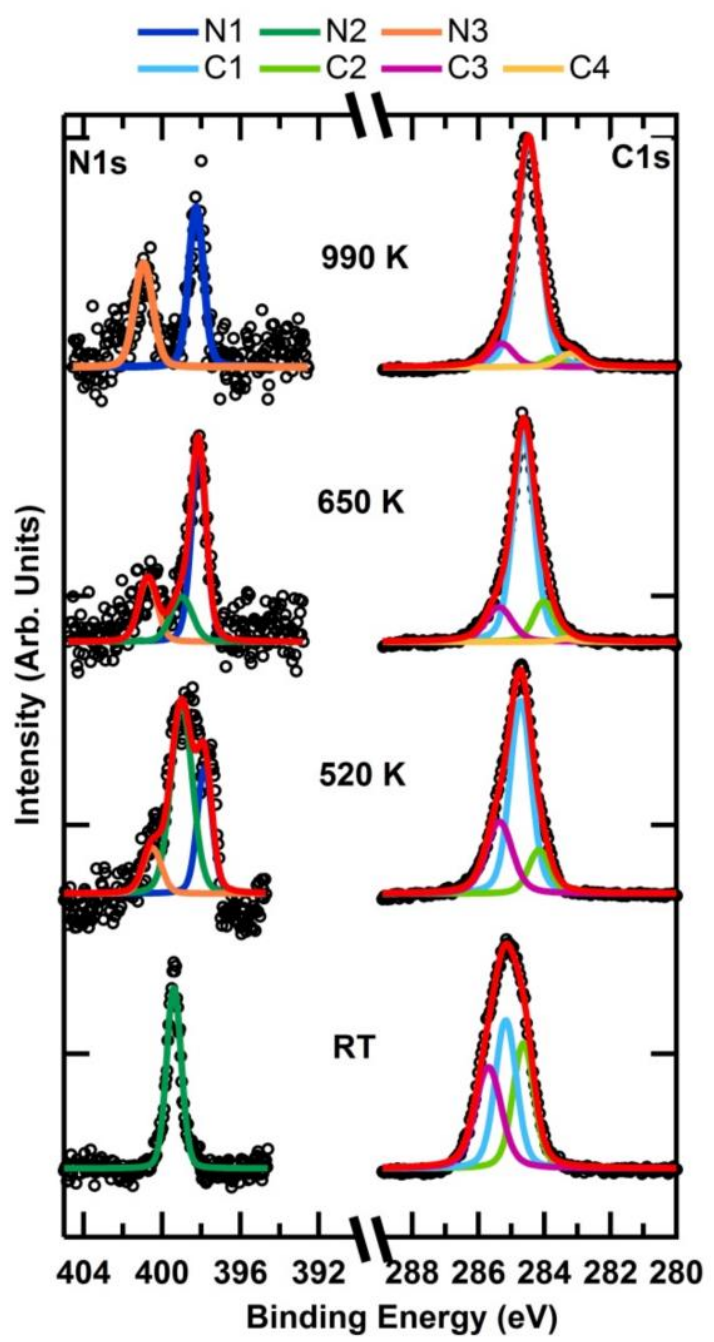

Figure 3. Evolution of the XPS spectra of submonolayer coverage of DiPy[5]DBH/Cu(110) with the temperature ranging from $R T$ to $990 K$. C1s XPS (right) has been fitted with four different components, whilst N1s (left) with three.

Remarkably, due to the high energy resolution of the beamline, we are able to follow the transformation of the molecule by XPS and confirm that the breakage of the $\mathrm{C}-\mathrm{H}$ bonds catalysed by the surface is the cause of the structural changes of the pristine molecules. The purple peak (C3) represents the carbon atoms linked to the $\mathrm{N}$ in the pyridine ring. ${ }^{20,37}$ This component still exists at $990 \mathrm{~K}$; therefore, the inclusion of $\mathrm{N}$ heteroatoms from the original precursor into the polymeric chains and graphene regions is quite efficient. The orange peak (C4) can be assigned to the $\mathrm{C}$ atoms of the molecules interacting with the Cu surface. ${ }^{38-39}$ This component is not present at room temperature, as the molecules diffuse and do not interact with the metal. This peak appears at about $650 \mathrm{~K}$, when polymeric networks are present, and its relative importance increases upon annealing. $\mathrm{DiPy}[5] \mathrm{DBH}$ on $\mathrm{Pt}(111)$ forms nanodomes, ${ }^{7}$ where 
only the $\mathrm{C}$ atoms at the edges are covalently bonded to the metal surface, and this model was also proposed by Lacovig et al. ${ }^{38}$ on similar systems; the same is probably happening here, where dehydrogenated $\mathrm{C}$ atoms which are not bonded to other molecules in the polymeric networks are bonded to the metal surface or the metal $(\mathrm{Cu})$ adatoms. The room temperature submonolayer N1s XPS of DiPy[5]DBH on Cu(110) (left panel of Figure 3) has only one narrow component at $399.4 \mathrm{eV}$ (dark green, N2). As both $\mathrm{N}$ atoms in the free molecules are pyridine-like $\mathrm{N}$ (the $\mathrm{N}$ bonded to two $\mathrm{C}$ atoms in an aromatic ring), the $\mathrm{N}$ component observed at $\mathrm{RT}$ is ascribed to the pyridinic bond. ${ }^{35,37}$ Upon annealing the N1s spectrum changes radically and two new components emerge and prevail, at around $398 \mathrm{eV}$ (dark blue, N1) and at around $400.6 \mathrm{eV}$ (orange, N3). As the oligomers and polymeric chains form, the molecule flattens, and the low BE blue peak (N1) begins to dominate. It represents $\mathrm{N}$ atoms in the pyridine ring belonging to the $\mathrm{N}$ doped nanographene flakes, that is, after the cyclodehydrogenation. ${ }^{35,40,41}$ These $\mathrm{N}$ atoms feel the $\mathrm{Cu}$ to a higher extent, because the pyridinic rings rotate upon the cleavage of $\mathrm{C}-\mathrm{H}$ bonds and the formation of new $\mathrm{C}-\mathrm{C}$ bonds, which brings the $\mathrm{N}$ and the $\mathrm{Cu}$ closer. The orange peak (N3) represents substitutional $\mathrm{N}$ atoms in the graphene network: these $\mathrm{N}$ atoms are bonded with three $\mathrm{sp}^{2}$ carbon atoms. ${ }^{35,37}$ This 'graphitic N' component is present already at $650 \mathrm{~K}$; however, STM images of the sample annealed at this temperature reveal the presence of polymeric chains, not of graphene regions, which will appear at a higher temperature. The presence of the substitutional $\mathrm{N}$ in the networks formed at $650 \mathrm{~K}$ indicates that some molecular chains bond via the pyridine rings, so that the linkage of building blocks in the chains can also involve the nitrogen atom binding with the carbon dangling bonds, hence enclosing an $N$ atom in the hexagonal carbon lattice substituting a $\mathrm{C}$ atom. A pictorial description of the carbon and nitrogen atoms configuration in relation with the XPS components analysed above is shown in Figure 4. NEXAFS and XPS measurements were performed for all the 3 systems: DiPy[5]DBH on $\mathrm{Cu}(110), \mathrm{Cu}(111)$ and $\mathrm{Au}(111)$ with analogous results, some of them already reported., ${ }^{1,42} \mathrm{We}$ selected the $\mathrm{Cu}(111)$ case for representing the NEXAFS and the $\mathrm{Cu}(110)$ for representing the XPS results because higher annealing temperatures have been reached for these substrates.

a) RT

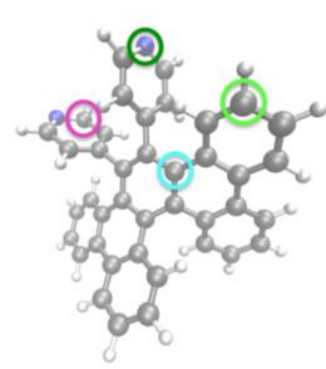

b) $990 \mathrm{~K}$

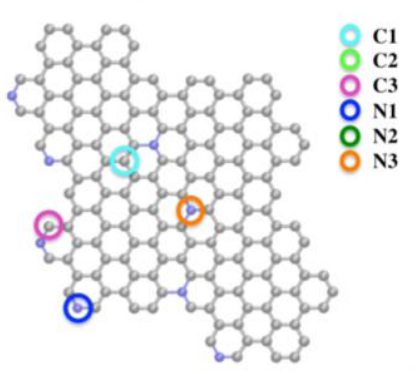

Figure 4. Schematic representation of the $\mathrm{N}$ and $\mathrm{C}$ atomic configuration related with the XPS peaks assignments of DiPy[5]DBH molecules on $\mathrm{Cu}(110)$. a) At RT (single molecule) and b) $990 \mathrm{~K}$ (nanographene flakes). C1 (light blue): $\mathrm{sp}^{2} \mathrm{C}$ atoms; $\mathrm{C} 2$ (light green): $\mathrm{C}-\mathrm{H}$; $\mathrm{C} 3$ (pink): $\mathrm{C}-\mathrm{N}$ pyridine ring; $\mathrm{C} 4$ : $\mathrm{C}-\mathrm{Cu}$, not represented; $\mathrm{N} 1$ (dark blue): $\mathrm{N}$ atoms in the pyridine ring interacting with $\mathrm{Cu}$; $\mathrm{N} 2$ (dark green): pyridine-like N; N3 (orange): substitutional N atoms in the graphene network.

To shed more light into how the temperature-induced nanostructure growth process is produced a series of DFT-based CI-NEB calculations have been carried out. Figure 5 summarizes the main results obtained from the simulations. Figure $5 a$ shows the unit cells used in the calculation of the optimized initial states of two partially dehydrogenated DiPy[5]DBH molecules on Cu(110), $\mathrm{Cu}(111)$ and $\mathrm{Au}(111)$. Figure $5 \mathrm{~b}$ shows top and side views of the optimized Initial State (IS), Transition State (TS) and Final State (FS) for the representative assembling process of two partially dehydrogenated DiPy[5]DBH molecules on $\mathrm{Cu}(110)$. We have chosen as starting point for all the three cases two DiPy[5]DBH molecules already partially dehydrogenated at the rim, which face each other. The choice of this particular partially dehydrogenated state in the molecules as starting point has been made on the basis of an already reported analysis, ${ }^{1}$ where 
the easiest-to-break $\mathrm{C}-\mathrm{H}$ bonds within the $\mathrm{DiPy}[5] \mathrm{DBH}$ molecule are those $\mathrm{C}-\mathrm{H}$ terminating bonds of the pyridinic rings. The molecules will exclusively react where they approach each other already dehydrogenated, otherwise, they will feel repulsion between them. This specific geometry for reaction is promoted in the experiment because the large diffusion and temperature, which make the molecules to shift rapidly from a site to the next. For all the three surfaces, the initial minimum distance between the two molecules (both starting from minimum energy on-surface sites) ranges between 3.5 and $4.5 \AA$. This starting geometrical configuration ensures that the MEPs will only describe the linking process between them. In Figure 5c we represent the total energy (referred to that of each initial state) as a function of the reaction coordinate along the computed MEP for $\mathrm{Au}(110), \mathrm{Cu}(111)$ and $\mathrm{Cu}(111)$ surfaces. The $\mathrm{CI}-\mathrm{NEB}$ calculations reveal, for all three surfaces, that the coupling process between the two partially dehydrogenated $\mathrm{DiPy}[5] \mathrm{DBH}$ molecules produces at moderately low transition state kinetic barriers of $0.22,0.37$ and $0.60 \mathrm{eV}$, yielding large assembling enthalpies of $10.4,12.2$ and 13.7 eV for $\mathrm{Au}(111), \mathrm{Cu}(111)$ and $\mathrm{Cu}(110)$, respectively.
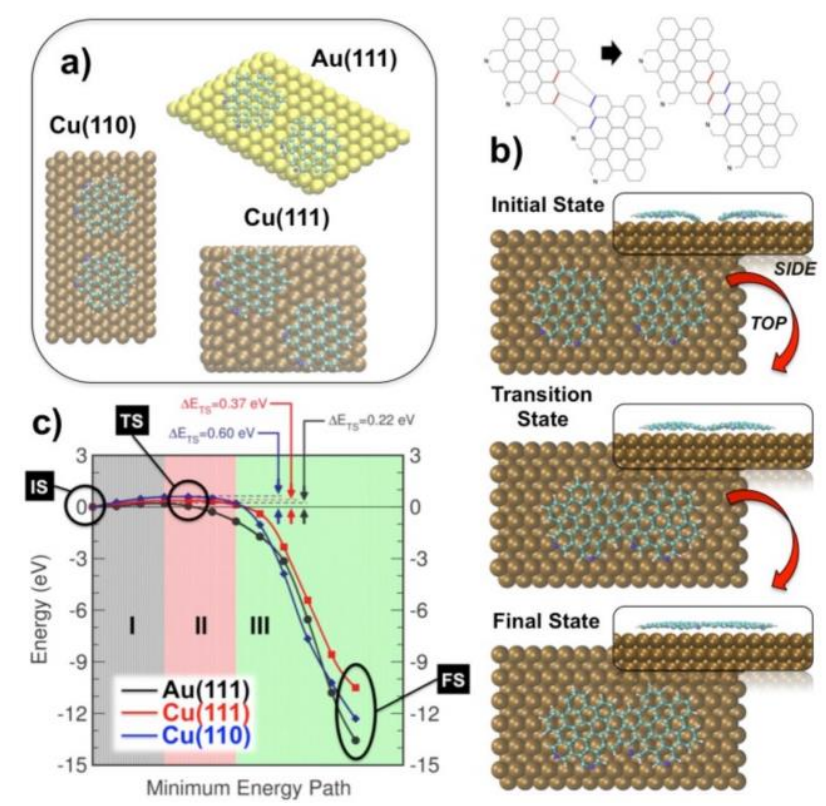

Figure 5. a) Unit cells used in the calculation of the optimized initial states of two partially dehydrogenated DiPy[5]DBH molecules on Cu(110), Cu(111) and Au(111). b) Top and side views of the optimized geometries of the most representative states (initial, transition and final states) for the assembling of two partially dehydrogenated DiPy[5]DBH molecules on the Cu(110) surface. c) Energy (in eV) as a function of the reaction coordinate along the computed CI-NEB Minimum Energy Path of the assembling process of two partially dehydrogenated DiPy[5]DBH molecules on $\mathrm{Au}(111)$ (black line), $\mathrm{Cu}(111)$ (red line) and $\mathrm{Cu}(110)$ (blue line). For a better comparison, in all cases all the energies have been referred to the corresponding initial state energies. Transition state energies, $\triangle E T S$, are also indicated in the figure for the three cases. Besides, three shading regions (named I, II and III) have been highlighted within the graph corresponding to the three different regimes along the three MEPS.

The difference between these values has its origin in the intrinsic reactivity of the different surfaces. ${ }^{43}$ In particular for the present case, on $\mathrm{Cu}(110)$ the linkage energy barrier adopts its higher value, since the $\mathrm{Cu}$ rows characterizing these surface are well-known enhanced reactivity points. This moderately high $\mathrm{Cu}(110)$ reactivity difficult the linkage process since the diffusing molecules experiments a higher barrier to fully detach the unsaturated $\mathrm{C}$ bonds from the surface before linking. Comparatively, this effect is substantially reduced for the case of $\mathrm{Cu}(111)$ surface (in around $0.23 \mathrm{eV}$ ), where these highly-reactive $\mathrm{Cu}$ rows are not present and the surface is totally flat. In this surface the fully detachment of the partially dehydrogenated linking molecules is easier, and the unsaturated edge C-bonds start to "feel" the unsaturated C-bonds of the incoming linking molecule at an early stage than for the case of $\mathrm{Cu}(111)$. Finally, the $\mathrm{Au}(111)$ is 
the less reactive surface, which is manifested by the fact that the barrier takes its lower value. For the three cases the assembling process is clearly exothermic and the formation of the macrostructure arising from the linkage of the two partially dehydrogenated molecules is very favourable. Figure $5 \mathrm{c}$ also shows three shading regions (named I, II and III) corresponding to the three different regimes observed along the MEPs. These three regions are perfectly identifiable in the morphology of all the MEP profiles and correspond to the following regimes:

i) Region I. Within this region of the MEPs the molecules start to get closer while they diffuse partially dehydrogenated on the surface (getting closer by around $0.2 \AA$ in each subsequent intermediate stage). The molecules start to feel the electronic states localized in the undersaturated dangling $\mathrm{C}$ atoms at the same time that they both undergo decoupling from the surface, which translates into a slight increase in the potential energy.

ii) Region II. The electronic states localized in the undersaturated dangling $\mathrm{C}$ atoms of both molecules start to hybridize, while these $\mathrm{C}$ atoms start to orientate to favour and maximize the electronic and structural coupling. An emerging competition between the molecule/surface and molecule/molecule interactions tends to slow down the increasing of the potential energy until reaching the transition state, where the energy vs. MEP profile finds its maximum.

iii) Region III. At this point both molecules show an almost complete decoupling from the surface, and the final linkage stage between them, driven by their dehydrogenated parts, tend to fully assemble both structures to saturate the dangling bonds created from the dehydrogenation. This makes that the MEP profiles decrease substantially the potential energy to deliver the whole linkage formation enthalpy. This behaviour is directly related not only with the diffusion ability of the molecules on the different surfaces, but also the temperature at which dehydrogenation occurs on these surfaces.

Our theoretical model misses several non-negligible factors such as other different linkage pathways, or an explicit treatment of the environmental temperature; however, the on-surface linkage between radicals occurs at moderately low energy barriers for the proposed models, and the interaction of the molecule with the surface, although exist (see Figure 5b), it is not strong enough to stop diffusion or inhibit coupling, as evinced by the experiments up to a certain temperature. Above this temperature, this bonding is responsible for the $\mathrm{C} 4$ component (Figure 3 right panel). Additionally, the CI-NEB calculations yield, for all three systems (involving more than 500 atoms), that the final nanostructures formed by the assembling process are almost flat, in agreement with the NEXAFS experiments. Thus, the present theoretical procedure, although simple by construction, provides valuable information on the energetics and kinetics in the formation of a final macrostructure from two partially dehydrogenated DiPy[5]DBH molecules as seed of the formation of larger nanoarchitectures.

\section{Conclusions}

We present a systematic spectroscopic and theoretical characterization of the process of thermally induced (cyclo)dehydrogenation of $\mathrm{DiPy}[5] \mathrm{DBH}$ on coinage metal surfaces to sequentially achieve the formation of hetero-aromatic polymeric networks and N-doped graphene flakes on different low-interacting metal surfaces. The combination of STM, XPS and NEXAFS at different annealing temperatures allow us to follow the transformations step by step. The behaviour of the pristine molecule upon annealing is very similar for the three coinage metal surfaces studied, namely $\mathrm{Cu}(110), \mathrm{Cu}(111)$ and $\mathrm{Au}(111)$. When the surface-assisted dehydrogenation begins, the molecules can meet and react with each other due to the diffusion upon deposition. The diffusing molecule presents a complex C1s XPS spectrum, as the contribution from graphitic $\mathrm{sp}^{2} \mathrm{C}$ atoms can be differentiated from the one of $\mathrm{C}$ atoms bonded to $\mathrm{H}$, and to $\mathrm{C}$ atoms in the pyridinic rings. The progressive loss in intensity of the $\mathrm{C}-\mathrm{H}$ component is a clear proof of the dehydrogenation upon annealing. The N1s XPS peak is present at all the annealing temperatures, hence confirming that all the structures formed (polymeric networks and graphene) are $\mathrm{N}$-doped and that this bottom-up approach is an effective way of controlling the doping of the resulting structures. The NEXAFS gives information on the geometry of the adsorbed molecule and it proves that the as-deposited molecules maintain their three- 
dimensional structure. As the molecules undergo both cyclodehydrogenation and dehydrogenative polymerisation upon annealing, they flatten and merge to form $\mathrm{N}$-doped nanostructures. Finally, DFT-based CI-NEB calculations provide valuable information on the assembly mechanism of the molecules on the way to larger nanoarchitectures.

\section{Acknowledgements}

This work has been supported by the Spanish MINECO (Grants MAT2011-26534 and MAT201454231-C04-1-P), the EU via the ERC-Synergy Program (Grant ERC-2013-SYG-610256 NANOCOSMOS) and the Innovation program under grant agreement No. 696656 (GrapheneCore1-Graphene-based disruptive technologies). JIM acknowledges the financial support by the "Ramón y Cajal" Program of MINECO (Grant RYC-2015-17730) and Nanocosmos, as well as computing resources from CTI-CSIC.

\section{Affiliations}

a. Materials Science Factory, Dept. Surfaces, Coatings and Molecular Astrophysics, Institute of Material Science of Madrid (ICMM-CSIC), C/Sor Juana Inés de la Cruz 3, 28049 Madrid, Spain.

b. MAX IV Laboratory, Lund University, Box 118, 22100 Lund, Sweden.

c. CNR-IOM, Laboratorio TASC, Basovizza SS-14, Km 163.5, 34149 Trieste, Italy.

d. Institute of Organic Chemistry and Biochemistry, Academy of Sciences of the Czech Republic, Flemingovo nám. 2, 16610 Prague, Czech Republic.

\section{Notes and references}

1 Pinardi, A. L.; Otero-Irurueta, G.; Palacio, I.; Martínez, J. I.; Sanchez-Sanchez, C.; Tello, M.; Rogero, C.; Cossaro, A.; Preobrajenski, A.; Lopez, M. F.; et al. Tailored Formation of N-Doped Nanoarchitectures by Diffusion-Controlled Dehydrogenation of Heteroaromatics. ACS Nano 2013, 7, 3676-3684.

2 Méndez, J.; López, M. F.; Martín-Gago, J. A. On-Surface Synthesis of Cyclic Organic Molecules. Chem. Soc. Rev. 2011, 40, 4578-90.

3 Lafferentz, L.; Eberhardt, V.; Dri, C.; Africh, C.; Comelli, G.; Esch, F.; Hecht, S.; Grill, L. Controlling on-Surface Polymerization by Hierarchical and Substrate-Directed Growth. Nat. Chem. 2012, 4, 215-20.

4 Treier, M.; Pignedoli, C. A.; Laino, T.; Rieger, R.; Müllen, K.; Passerone, D.; Fasel, R. SurfaceAssisted Cyclodehydrogenation Provides a Synthetic Route towards Easily Processable and Chemically Tailored Nanographenes. Nat. Chem. 2011, 3, 61-7.

5 Otero, G.; Biddau, G.; Sánchez-Sánchez, C.; Caillard, R.; López, M. F.; Rogero, C.; Palomares, F. J.; Cabello, N.; Basanta, M. A.; Ortega, J.; et al. Fullerenes from Aromatic Precursors by Surface-Catalysed Cyclodehydrogenation. Nature 2008, 454, 865-869.

6 Talirz, L.; Ruffieux, P; Fasel, R., On-Surface Synthesis of Atomically Precise Graphene Nanoribbons. Adv. Mate. 2016, 28, 6222-6231.

7 Pinardi, A. L.; Martínez, J. I.; Jancarik, A.; Stara, I. G.; Stary, I. ; Lopez, M. F.; Méndez, J.; Martin-Gago, J. A. Sequential Formation of $\mathrm{N}$-doped Nanohelicenes, Nanographenes and Nanodomes by Surface-Assisted Chemical (Cyclo)dehydrogenation of Heteroaromatics. Chem. Commun. 2014, 50, 1555-1557.

8 Shuler, B.; Collazos, S.; Gross, L.; Meyer, G.; Pérez, D.; Guitián, E.; Peña, D. From Perylene to a 22-ring Ảromatic Hydrocarbon in One-Pot. Ảngew. Chem. 2014, 126, 9150.

9 Floreano, L.; Naletto, G.; Cvetko, D.; Gotter, R.; Malvezzi, M.; Marassi, L.; Morgante, A.; Santaniello, A.; Verdini, a.; Tommasini, F.; Tondello, G. Rev. Sci. Instrum. 1999, 70, 3855.

10Baroni, S.; Corso, A. D.; de Gironcoli, S.; Giannozzi, P. QUANTUM ESPRESSO Package; 2005.

11 Grimme, S. Semiempirical GGA-type Density Functional Constructed with a Long-range Dispersion Correction. J. Comput. Chem. 2006, 27, 1787-1799.

12 Barone, V.; Casarin, M.; Forrer, D.; Pavone, M.; Sambi M.; Vittadini, A. Role and Effective Treatment of Dispersive Forces in Materials: Polyethylene and Graphite Crystals as Test Cases. J. Comp. Chem. 2009, 30, 934-939.

13 Vanderbilt, D. Soft Self-consistent Pseudopotentials in a Generalized Eigenvalue Formalism. Phys. Rev. B. 1990, 41, 7892-7895.

14 Perdew, J. P.; Burke, K.; Ernzerhof, M. Generalized Gradient Approximation Made Simple. Phys. Rev. Lett. 1996, 77, 3865-3868.

15Monkhorst, H. J.; Pack. J. D. Special Points for Brillouin-zone Integrations. Phys. Rev. B 1976, 13, 5188-5192.

16 Methfessel, M.; Paxton, A. T. High-precision Sampling for Brillouin-zone Integration in Metals. Phys. Rev. B 1989, 40, 3616-3621. 
17 Classical and Quantum Dynamics in Condensed Phase Simulations; Berne, B. J., Cicotti, G., Coker, D. F., Eds.; World Scientific Publishing Company: Singapore, 1998.

18 Henkelman, G.; Jónsson, $H$. Improved Tangent Estimate in the Nudged Elastic Band Method for Finding Minimum Energy Paths and Saddle Points. J. Chem. Phys. 2000, 113, 9978-9985.

19 Henkelman, G.; Uberuaga, B. P.; Jónsson, H. A Climbing Image Nudged Elastic Band Method for Finding Saddle Points and Minimum Energy Paths. J. Chem. Phys. 2000, 113, 9901-9904

20 Hellgren, N.; Guo, J.; Luo, Y.; Såthe, C.; Agui, A.; Kashtanov, S.; Nordgren, J.; Agren, H.; Sundgren, J.-E. Electronic Structure of Carbon Nitride Thin Films Studied by X-ray Spectroscopy Techniques. Thin Solid Films 2005, 471, 19-34.

21 Kolczewski C.; Püttner R.; Martins M.; Schlachter A. S.; Snell G.; Sant'Anna M. M.; Hermann K.; Kaindl G. Spectroscopic analysis of small organic molecules: A comprehensive near-edge x-rayabsorption fine-structure study of C6-ring-containing molecules. J. Chem. Phys. 2006, 124, 034302.

22 Preobrajenski, A. B.; Ng, M. L.; Vinogradov, A. S.; Mårtensson, N. Controlling Graphene Corrugation on Lattice-Mismatched Substrates. Phys. Rev. B 2008, 78, 2-5.

$23 \mathrm{Ng}$, M. L.; Balog, R.; Hornekær, L.; Preobrajenski, A. B.; Vinogradov, N. A.; Mårtensson, N.; Schulte, K. Controlling Hydrogenation of Graphene on Transition Metals. J.' Phys. Chem. C 2010, 114, 18559-18565.

24 Oji, H.; Mitsumoto, R.; Ito, E.; Ishii, H.; Ouchi, Y.; Seki, K.; Yokoyama, T.; Ohta, T.; Kosugi, N. Core Hole Effect in NEXAFS Spectroscopy of 'Polycyclic Aromatic Hydrocarbons: Benzene, Chrysene, Perylene, and Coronene. J. Chem. Phys. 1998, 109, 10409-10418.

25Zhang, W.; Nefedov, A.; Naboka, M.; Cao, L.; Wöll, C. Molecular Orientation of Terephthalic Acid Assembly on Epitaxial Graphene: NEXAFS and XPS Study. Phys. Chem. Chem. Phys. 2012, 14, 10125-31.

26 Beernink, G.; Gunia, M.; Dötz, F.; Öström, H.; Weiss, K.; Müllen, K.; Wöll, C. Synthesis of Polycyclic Aromatic Hydrocarbons and Graphite Islands via Surface-Induced Reaction of Small Molecules. ChemPhysChem 2001, 2, 317-320.

27 Lee, V.; Park, C.; Jaye, C.; Fischer, D. A.; Yu, Q.; Wu, W.; Liu, Z.; Bao, J.; Pei, S.-S.; Smith, C.; Lysaght, P.; Banerjee, S. Substrate Hybridization and Rippling of Graphene Evidenced by NearEdge X-ray Absorption Fine Structure Spectroscopy. J. Phys. Chem. Lett. 2010, 1, 1247-1253.

28 Schultz, B. J.; Patridge, C. J.; Lee, V.; Jaye, C.; Lysaght, P. S.; Smith, C.; Barnett, J.; Fischer, D. A.; Prendergast, D.; Banerjee, S. Imaging Local Electronic Corrugations and Doped Regions in Graphene. Nat. Commun. 2011, 2, 372.

29 Hähner, G. Near Edge X-ray Absorption Fine Structure Spectroscopy as a Tool to Probe Electronic and Structural Properties of Thin Organic Films and Liquids. Chem. Soc. Rev. 2006, 35, 1244-55.

30 Urquhart, S. G.; Gillies, R. Rydberg-Valence Mixing in the Carbon 1s Near-Edge X-ray Absorption Fine Structure Spectra of Gaseous Alkanes. J. Phys. Chem. A 2005, 109, 2151-9.

31 Püttner R.; Kolczewski C.; Martins M.; Schlachter A. S.; Snell G.; Sant'Anna M. M.; Viefhaus J.; Hermann K.; Kaindl G. The C1s NEXAFS spectrum of benzene below threshold: Rydberg or valence character of the unoccupied $\sigma$-type orbitals. Chem. Phys. Lett. 2004, 393, 361.

32 Dedkov, Y. S.; Sicot, M.; Fonin, M. X-ray Absorption and Magnetic Circular Dichroism of Graphene/Ni(111). J. Appl.' Phys. 2010, 107, 09E121.

33 El-Sayed, A.; Mowbray, D. J.; García-Lastra, J. M.; Rogero, C.; Goiri, E.; Borghetti, P.; Turak, A.; Doyle, B. P.; Dell'Angela, M.; Floreano, L.; et al. Supramolecular Environment-Dependent Electronic Properties of Metal-Organic Interfaces. J. Phys. Chem. C 2012, 116, 4780-4785.

34 Baldacchini, C.; Allegretti, F.; Gunnella, R.; Betti, M. G. Molecule-Metal Interaction of Pentacene on Copper Vicinal Surfaces. Surf. Sći. 2007, 601, 2603-2606.

35 Usachov, D.; Vilkov, O.; Grüneis, A.; Haberer, D.; Fedorov, A.; Adamchuk, V. K.; Preobrajenski, A. B.; Dudin, P.; Barinov, A.; Oehzelt, M.; et al. Nitrogen-Doped Graphene: Efficient Growth, Structure, and Electronic Properties. Nano Lett. 2011, 11, 5401-7.

36 Gustafsson, J.; Zhang, H.; Moons, E.; Johansson, L. Electron Spectroscopy Studies of PTCDA on AgSi(111)-3×3. Phys. Rev. B 2007, 75, 155413-155419.

$37 \mathrm{Lv}$, R.; Li, Q.; Botello-Méndez, A. R.; Hayashi, T.; Wang, B.; Berkdemir, A.; Hao, Q.; Elías, A. L.; Cruz-Silva, R.; Gutiérrez, H. R.; et al. Nitrogen-Doped Graphene: Beyond Single Substitution and Enhanced Molecular Sensing. Sci. Rep. 2012, 2, 1-8.

38 Lacovig, P.; Pozzo, M.; Alfè, D.; Vilmercati, P.; Baraldi, A.; Lizzit, S. Growth of Dome-Shaped Carbon Nanoislands on $\operatorname{Ir}(111)$ : The Intermediate between Carbidic Clusters and Quasi-FreeStanding Graphene. Phys. Rev. Lett. 2009, 103, 14-17.

39 Grüneis, A.; Kummer, K.; Vyalikh, D. V Dynamics of Graphene Growth on a Metal Surface: a Time-Dependent Photoemission Study. New J. Phys. 2009, 11, 073050.

40 Kang, X.; Song, Y.; Chen, S. Nitrene-Functionalized Ruthenium Nanoparticles. J. Mater. Chem. 2012, 22, 19250 .

41 Soto, G. Synthesis of PtNx Films by Reactive Laser Ablation. Mater. Lett. 2004, 58, 2178-2180.

42 Martín-Gago, J. A.; Pinardi, A. L.; Martínez, J. I. "On-surface (Cyclo-)dehydrogenation Reactions: Role of Surface Difussion". In book: On-surface Synthesis. Springer Series "Advances in Atom and Molecule Machines" edited by André Gourdon. Chapter 3, pp. 43-83. Springer International Publishing Switzerland, 2016. 
43Duhm S. Gerlach A. Salzmann I. Bröker B. Johnson R.L. Schreiber F. and Koch N. PTCDA on $\mathrm{Au}\left(\begin{array}{ll}1 & 1\end{array}\right), \mathrm{Ag}\left(\begin{array}{lll}1 & 1\end{array}\right)$ and $\mathrm{Cu}\left(\begin{array}{ll}1 & 1\end{array}\right)$ : Correlation of interface charge transfer to bonding distance. Org. Electron. 2008, 9, 111-118. 